\title{
ON THE OPTIMAL CONTROL OF SINGLE-STAGE HYBRID MANUFACTURING SYSTEMS VIA NOVEL AND DIFFERENT VARIANTS OF PARTICLE SWARM OPTIMIZATION ALGORITHM
}

\author{
M. SENTHIL ARUMUGAM AND M. V. C. RAO
}

Received 1 March 2005

This paper presents several novel approaches of particle swarm optimization (PSO) algorithm with new particle velocity equations and three variants of inertia weight to solve the optimal control problem of a class of hybrid systems, which are motivated by the structure of manufacturing environments that integrate process and optimal control. In the proposed PSO algorithm, the particle velocities are conceptualized with the local best (or pbest) and global best (or gbest) of the swarm, which makes a quick decision to direct the search towards the optimal (fitness) solution. The inertia weight of the proposed methods is also described as a function of pbest and gbest, which allows the PSO to converge faster with accuracy. A typical numerical example of the optimal control problem is included to analyse the efficacy and validity of the proposed algorithms. Several statistical analyses including hypothesis test are done to compare the validity of the proposed algorithms with the existing PSO technique, which adopts linearly decreasing inertia weight. The results clearly demonstrate that the proposed PSO approaches not only improve the quality but also are more efficient in converging to the optimal value faster.

\section{Introduction}

The hybrid systems combine two different types of categories, subsystems with continuous dynamics and subsystems with discrete dynamics that interact with each other. Such hybrid systems arise in varied contexts in manufacturing, communication networks, automotive engine design, computer synchronization, and chemical processes, among others. In hybrid manufacturing systems, which is considered in this paper, the manufacturing process is composed of the event-driven dynamics of the parts moving among different machines and the time-driven dynamics of the processes within particular machines. Frequently in hybrid systems, the event-driven dynamics are studied separately from the time-driven dynamics, the former via timed state automata or Petri net models, PLC etc., and the latter via differential or difference equations [6].

The hybrid framework can be modeled either by extending the event-driven models with time-driven dynamics; or by extending the traditional time-driven models with event- driven dynamics. The hybrid system-modeling framework, which is motivated by 


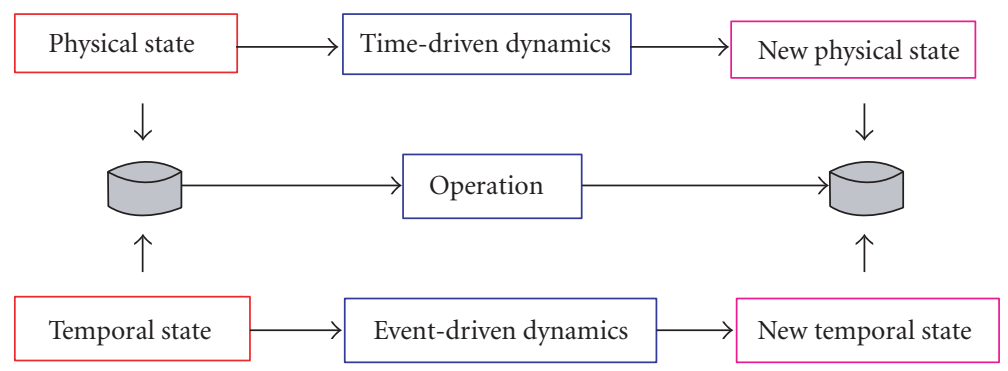

FIGURE 2.1. Hybrid framework with time-driven and event-driven dynamics.

the structure of many manufacturing system, considered in this research adopts the first category. To represent the hybrid nature of the model, each job is characterized by a physical state and a temporal state. The physical state represents the physical characteristics of interest and evolves according to the time-driven dynamics (e.g., difference or differential equations) while the job is being processed by a server. The temporal state represents processing arrival and completion times and evolves according to the discrete-event dynamics (e.g., queuing dynamics). The interaction of time-driven with event-driven dynamics leads to a natural tradeoff between temporal requirements on job completion times and physical requirements on the quality of the completed jobs (see Figure 2.1). Such modeling frameworks and optimal control problems have been considered in $[1,8]$.

A number of algorithms were developed so far to solve such optimal control problems. Particle swarm optimization (PSO) is one of the modern heuristic algorithms under the evolutionary algorithms (EA) and gained lots of attention in solving optimal control problems. Several variants of the PSO technique have been proposed so far, following Eberhart and Kennedy [3, 4]. In this paper, different global versions of PSO with modified velocity equations and inertia weights are investigated. The parameter selections in the PSO equations are carefully anlaysed in terms of pbest and gbest. Three different inertia weight (one standard and two new) variants are adopted with four versions (one existing and three proposed) of velocity equations are investigated in this paper. Among such 12 methods, the best methods are identified and their validity is verified through a number of statistical analyses and approaches.

The remaining of this paper is organized as follows: In Section 2, the optimal control problem of a single-stage hybrid manufacturing system is studied and formulated. The functional procedure and behavior of standard PSO are briefed in Section 3. Section 4 depicts the design of new inertia weight variants, modified velocity equations and the parameter selections for PSO algorithms. The numerical example, the simulation results and the statistical analyses are given in Section 5 and finally the discussions and conclusions are drawn in Section 6.

\section{Problem formulation of single-stage hybrid manufacturing system}

The general hybrid system framework with event-driven and time-driven dynamics is given in Figure 2.1. 


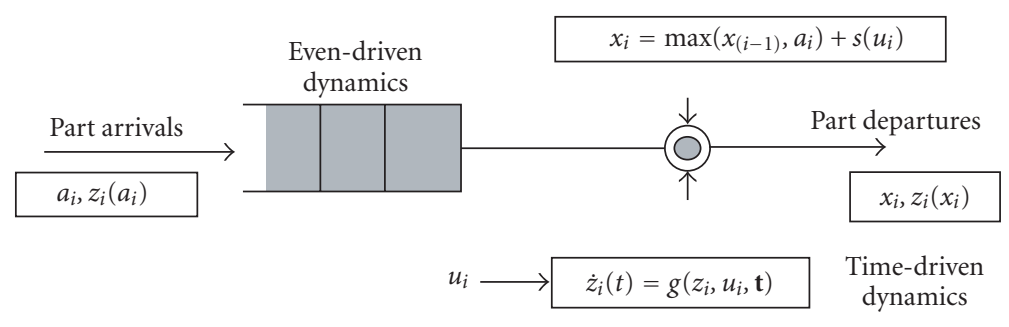

FIgURe 2.2. A single-stage hybrid manufacturing system.

Consider the hybrid model of a single-stage manufacturing hybrid system model is shown in Figure 2.2. A sequence of $N$ jobs $\left(C_{1}, C_{2}, C_{3}, \ldots, C_{N}\right)$ is assigned by an external source to arrive for processing at known times $0 \leq a_{1} \leq a_{2} \leq \cdots \leq a_{N}$. The jobs are processed first-come first-serve (FCFS) basis by a work-conserving and nonpreemptive server. The processing time is $s\left(u_{i}\right)$, which is a function of a control variable $u_{i}$, and $s\left(u_{i}\right) \geq 0$.

The time-driven dynamics of the hybrid system is defined by the equation which evolved the job $C_{i}$ which is initially at some physical state $\xi_{i}$ at time $x_{0}$.

$$
\dot{z}_{i}(t)=g\left(z_{i}, u_{i}, t\right), \quad z_{i}\left(x_{0}\right)=\xi_{i}
$$

The event-driven dynamics is described by recursive non-linear equations (Max-plus equations) involving a maximum or a minimum operation, which is typically found in models of discrete event systems (DES). For the fist-come first-serve (FCFS), nonpreemptive, single server example in Figure 2.2, these dynamics are given by the "max-plus" recursive equation

$$
x_{i}=\max \left(x_{(i-1)}, a_{i}\right)+s\left(u_{i}\right), \quad i=1, \ldots, N,
$$

where $x_{i}$ is the departure or completion time of $i$ th job and $x_{0}=-\infty$. The recursive relationship given in (2.2) is known in queuing theory as the Lindley equation [8].

From (2.1) and (2.2), it is clear that the choice of control $u_{i}$ affects both the physical state $z_{i}$ and next temporal state $x_{i}$, and thus time-driven dynamics (2.1) and event-driven dynamics (2.2), justifying the hybrid nature of the system. According to [6], there are two alternative ways to view this hybrid system. The first is as a discrete event queuing system with time-driven dynamics evolving during processing in the server as shown in Figure 2.3.

The second viewpoint interprets the model as a switched system. In this framework, each job must be processed until it reaches a certain "quality level" denoted by $\Gamma_{i}$ (e.g., a threshold above which $z_{i}$ satisfies a desired quality level). That is, the processing time for 


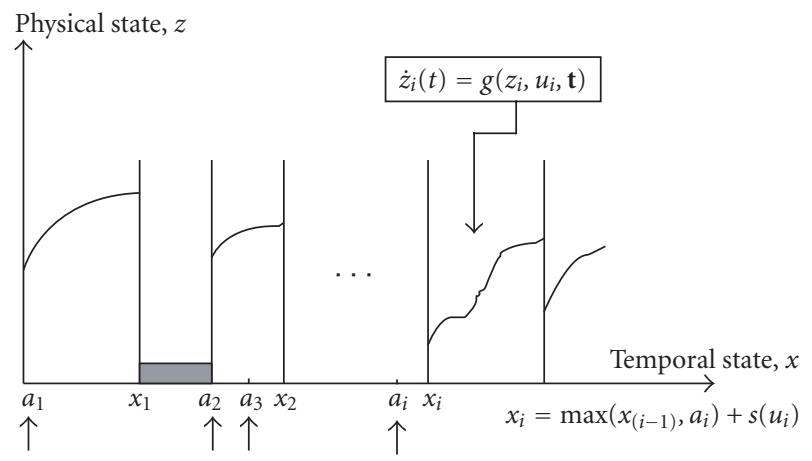

Figure 2.3. Typical trajectory.

each job is chosen to satisfy the stopping rule

$$
s_{i}\left(u_{i}\right)=\min \left[t \geq 0 ; z_{i}\left(t_{0}\right)=\int_{t_{0}}^{t_{0}+t} g_{i}\left(\tau, u_{i}, t\right) d \tau+z\left(t_{0}\right) \in \Gamma_{i}\right] .
$$

Figure 2.3 shows the evolution of the physical state as a function of time $t$. It is shown in the figure that the dynamics of the physical state experiences a "switch" when certain events occur. These events may classify into two categories: uncontrolled (or exogenous) arrival events and controlled departure events. In Figure 2.2, the first event is an exogenous arrival event at time $a_{1}$. When the first job arrives at $a_{1}$, the physical state starts to evaluate the time-driven dynamics until it reaches the departure time $x_{1}$. It is clearly observed that the first job completes before the second job arrives and hence there is an idle period, in which the server has no jobs to process. The physical state again begins evolving the time-driven dynamics at time $a_{2}$ (arrival of second job) until the second job completes at $x_{2}$. However, that the third job arrived before the second job was completed. So the third job is forced to wait in the queue until time $x_{2}$. After the second job completes at $x_{2}$ the physical state begins to process the third job. As indicated in Figure 2.3, not only do the arrival time and departure time cause switching in the time-driven dynamics according to (2.1), but also the sequence in which these events occur is governed by the event-driven dynamics given in (2.2).

This system is hybrid is the sense that it combines the time-driven dynamics (2.1) with the event-driven dynamics (2.2), the two being coupled through the choice of control sequence $\left\{u_{1}, \ldots, u_{N}\right\}$. Hence the optimal control problem considered in this paper is to minimize an objective function of the form

$$
J=\sum_{i=1}^{N}\left\{\theta_{i}\left(u_{i}\right)+\phi_{i}\left(x_{i}\right)\right\}^{\eta} .
$$

Although, in general, the state variables $z_{i}, \ldots, z_{N}$ evolve continuously with time, minimizing (2.4) is an optimization problem in which the values of the state variables are considered only at the job completion times $x_{1}, \ldots, x_{N}$. Since the stopping criterion in (2.3) is used to obtain the service times, a cost on the physical state $z_{i}\left(x_{i}\right)$ is unnecessary 
because the physical state of each completed job satisfies the quality objectives, that is, $z_{i}\left(x_{i}\right) \in \Gamma_{i}$.

Obviously, the control variable $u_{i}$ is affecting the processing time through $s_{i}=s\left(u_{i}\right)$ for extensions to cases with time-varying controls $u_{i}(t)$ over a service time. By assuming $s_{i}(\cdot)$ is either monotone increasing or monotone decreasing, given a control $u_{i}$, service time $s_{i}$ can be determined from $s_{i}=s\left(u_{i}\right)$ and vice versa. For simplicity, let $s_{i}=u_{i}$ and the rest of the analysis is carried out with the notation $u_{i}$. Hence the optimal control problem, with $\eta=1.5$, denoted by $P$ is of the following form:

$$
P: \min _{u_{1}, \ldots, u_{N}}\left\{J=\sum_{i=1}^{N}\left\{\theta_{i}\left(u_{i}\right)+\phi_{i}\left(x_{i}\right)\right\}^{1.5}: u \geq 0, i=1, \ldots, N\right\}
$$

subject to

$$
x_{i}=\max \left(x_{(i-1)}, a_{i}\right)+s\left(u_{i}\right), \quad i=1, \ldots, N .
$$

The optimal solution of $P$ is denoted by $u_{i}^{*}$ for $i=1, \ldots, N$, and the corresponding departure time in (2.6) is denoted by $x_{i}^{*}$ for $i=1, \ldots, N$.

\section{Review of standard particle swarm optimization techniques}

The particle swarm optimization (PSO) is a parallel evolutionary computation technique developed by Kennedy and Eberhart based on the social behavior of metaphor. The PSO technique has ever since turned out to be a competitor in the fields of numerical optimization. The evolutionary algorithms, EAs, like genetic algorithm (GA) and evolutionary programming (EP) are search algorithms based on the simulated evolutionary process of natural selection, variation, and genetics. Both GA and EP can provide a near global solution [4]. PSO is similar to the other evolutionary algorithms in that the system is initialized with a population of random solutions, conceptualized as particle. Each particle is assigned a randomized velocity and is iteratively moved through the problem space. It is attracted towards the location of the best fitness achieved so far by the particle itself, called as personal best (pbest) and the location of the best fitness achieved so far across the whole population, known as global best (gbest). The PSO algorithm includes some tuning parameters which are clearly influence the performance of the algorithm, often referred to as exploration-exploitation tradeoff. Exploration is the ability to test various regions in the problem space in order to locate a good optimum, hopefully a global solution. Exploitation is the ability to concentrate the search around a promising candidate solution in order to locate the optimum precisely. A complete theoretical analysis of PSO has been described by Clerc and Kennedy in [2].

Also, PSO will not follow survival of the fittest, the principle of other EAs. PSO when compared to EP has very fast converging characteristics; however it has a slow fine-tuning ability of the solution. Also PSO has a more global searching ability at the beginning of the run and a local search near the end of the run. Therefore, while solving problems with more local optima, there are more possibilities for the PSO to explore local optima at the end of the run $[5,7]$. 
PSO is basically through simulation of bird flocking in two-dimension space. The position of each particle is represented by XY axis position and the velocity is expressed by $v x$ (the velocity of $\mathrm{X}$ axis) and $v y$ (the velocity of $\mathrm{Y}$ axis). Modification of the particle position is realized by position and velocity information. Each particle knows its best value so far (pbest) and its XY position. The information corresponds to personal experiences of each particle in the concept of individual learning and cultural transmission (ILCT). Moreover, each particle knows the best value so far in the group (gbest) among pbests. The information corresponds with the knowledge of how the other particles around them have performed in the concept of ILCT [5]. Namely, each particle tries to modify its position using the following information:

(i) the distance between the current position and pbest;

(ii) the distance between the current position and gbest.

This modification can be represented by the concept of velocity. Velocity of each particle can be modified by the following equation:

$$
v_{i}^{k+1}=w v_{i}^{k}+c_{1} \text { rand }_{1} \times\left(\text { pbest }_{i}-X_{i}^{k}\right)+c_{2} \text { rand }_{2} \times\left(\text { gbest }-X_{i}^{k}\right),
$$

where

(i) $v_{i}^{k+1}$ : velocity of particle $i$ at iteration $k$;

(ii) $w$ : weighting function;

(iii) $c_{1}$ and $c_{2}$ : two positive constants named as cognitive and social parameter respectively (normally $c_{1}=c_{2}=2$ );

(iv) rand: random number between 0 and 1 ;

(v) $X_{i}^{k}$ : current position of particle $i$ at iteration $k$;

(vi) pbest $t_{i}$ pbest of particle $i$;

(vii) gbest: gbest of the group.

And the current position can be modified by the following equation:

$$
X_{i}^{k+1}=X_{i}^{k}+v_{i}^{k+1}
$$

In the standard PSO, the inertia weight is introduced as a decreasing function which is set to a higher value $\left(w_{\max }\right)$ at initial stage and is decreased linearly with the iteration, $k$ to a lower value $\left(w_{\min }\right)$ and it is represented by the equation

$$
w=w_{\max }-\left(\frac{w_{\max }-w_{\min }}{k_{\max }}\right) \times k,
$$

where $k_{\max }$ is the maximum iteration number.

From (3.1), three terms are taken into consideration: the first term is $w v_{i}^{k}$, is the particle's previous velocity weighted by the inertia weight $w$. The second term, $\left(\right.$ pbest $\left._{i}-X_{i}^{k}\right)$, 


\section{TABLE 4.1}

\begin{tabular}{|c|c|c|}
\hline Method & PSO parameter & Velocity equation \\
\hline $\mathrm{W}$ & Standard PSO & $v_{i}^{k+1}=w v_{i}^{k}+c_{1}$ rand $_{1} \times\left(\right.$ pbest $\left._{i}-X_{i}^{k}\right)+c_{2}$ rand $_{2} \times\left(\right.$ gbest $\left.-X_{i}^{k}\right)$ \\
\hline \multirow[t]{2}{*}{ X } & $c_{1}=c_{2}=\left(1+\frac{\text { pbest }_{i}}{\text { gbest }}\right)$ & $v_{i}^{k+1}=w v_{i}^{k}+\left(1+\frac{\text { pbest }}{g_{i} \text { best }}\right) \times$ rand $\times\left(\right.$ pbest $_{i}+$ gbest $\left.-2 X_{i}^{k}\right)$ \\
\hline & $\operatorname{rand}_{1}=\operatorname{rand}_{2}=$ rand & \\
\hline $\mathrm{Y}$ & $c_{1}=c_{2}=2$ & $v_{i}^{k+1}=w v_{i}^{k}+\left[2 \times\right.$ rand $\times\left(\right.$ pbest $_{i}+$ gbest $\left.\left.-2 X_{i}^{k}\right)\right]$ \\
\hline \multirow[t]{2}{*}{ Z } & $\begin{array}{l}\operatorname{rand}_{1}=\text { rand }_{2} \\
c_{1}=c_{2}=\left(1+\frac{\text { gbest }}{\text { pbest }_{i}}\right)\end{array}$ & $v_{i}^{k+1}=w v_{i}^{k}+\left(1+\frac{\text { gbest }}{\text { pbest }_{i}}\right) \times$ rand $\times\left(\right.$ pbest $_{i}+$ gbest $\left.-2 X_{i}^{k}\right)$ \\
\hline & $\operatorname{rand}_{1}=\operatorname{rand}_{2}=$ rand & \\
\hline
\end{tabular}

TABLE 4.2

\begin{tabular}{cl}
\hline Method & Inertia weight \\
\hline $\mathrm{A}$ & $w=w_{\max }-\left(\frac{w_{\max }-w_{\min }}{k_{\max }}\right) \times k$ \\
$\mathrm{~B}$ & $w_{i}=\left(1-\frac{g^{\text {best }}}{\text { pbest }_{i}}\right)$ \\
$\mathrm{C}$ & $w_{i}=\left(1-\frac{\text { gbest }_{i}}{\left(\text { pbest }_{i}\right)_{\text {average }}}\right)$ \\
\hline
\end{tabular}

is the distance between the particle's best previous position, and its current position. And the third term, $\left(\right.$ gbest $\left.-X_{i}^{k}\right)$, is the distance between the swarm's best experience, and the $i$ th particle's current position. Equation (3.2) provides the new position of $i$ th particle, adding its new velocity, to its current position. In general, the performance of each particle is measured according to a fitness function, which is problem-dependent. In optimization problems, the fitness function is usually the objective function under consideration.

\section{New variants of inertia weight and velocity equation}

The role of inertia weight is very crucial on PSO's performance and convergence behavior. The inertia weight is employed to control the impact of the history of velocities on the current velocity. In this way, the inertia weight regulates the trade-off between global and local exploration abilities of the swarm. A suitable value for the inertia weight provides a balance between the global and local exploration ability of the swarm which results in better convergence rates. Similarly, the velocity equation of PSO also plays an important role for a quality solution and faster convergence. Since the new position of the particle depends on the velocity, it is very important to design the parameters in the velocity equation carefully in order to move closer to the optimal solution faster. Such a careful design of PSO parameters is considered in this paper. In this paper, new variants to the inertia weight and velocity equations are considered and they are classified into two groups. Group 1 (Table 4.1) includes the new proposed velocity equations and Group 2 (Table 4.2) consists of inertia weight variants. 
Step 1. Initialize

Set index of Global best (Gbest index) $=1$.

Step 2. Create population

Randomize the positions and velocities for entire population

Set the reference value of best position PB (i). Fitness

Update velocity vector.

Step 3. Calculate $\mathrm{P}(\mathrm{i})$. Fitness

If $\mathrm{P}$ (i). Fitness $<$ reference value of best position $\mathrm{PB}$ (i). Fitness Then

Set new reference value of best position as $\mathrm{P}(\mathrm{i})$. Fitness

If $\mathrm{PB}$ (i). Fitness < PB (GBestIndex). Fitness

Then Set new GBestIndex $=i$.

Step 4. Calculate the particle velocity using the swarm equations given in group $1 \& 2$

Update the particle positions using (3.1).

Step 5. Repeat until Max number of generation or best solution.

Algorithm 4.1

Each method described in group 1 adopts all the three inertia weights given in group 2 individually and hence 12 methods are formed namely methods WA, WB, WC, XA, XB, $\mathrm{XC}, \mathrm{Y}, \mathrm{YB}, \mathrm{YC}, \mathrm{ZA}, \mathrm{ZB}$, and ZC. All these 12 methods are implemented in the PSO algorithm (Algorithm 4.1), to solve the optimal control problem of the single-stage hybrid manufacturing systems.

\section{Experimental results and statistical analyses}

In order to compare the validity and usefulness of the proposed PSO methods, the optimal control problem from (2.5) and (2.6) with the following functions are considered:

$$
\theta_{i}\left(u_{i}\right)=\frac{2}{u_{i}}, \quad \phi\left(x_{i}\right)=3^{*} x_{i}, \quad \eta=1.5
$$

Now (2.5) becomes

$$
\min _{u_{1}, \ldots, u_{N}}\left\{J=\sum_{i=1}^{N}\left(\frac{2}{u_{i}}+3 x_{i}\right)^{1.5}\right\}
$$

subject to

$$
x_{i}=\max \left(x_{(i-1)}, a_{i}\right)+u_{i} .
$$

The optimal controls $\left(u_{i}\right)$, the departure time $\left(x_{i}\right)$ and cost or fitness $(J)$ for the objective function given in (5.2) are computed with the following parameter settings.

(i) The maximum number of generations is set as 2000 .

(ii) The population size $=20$. 


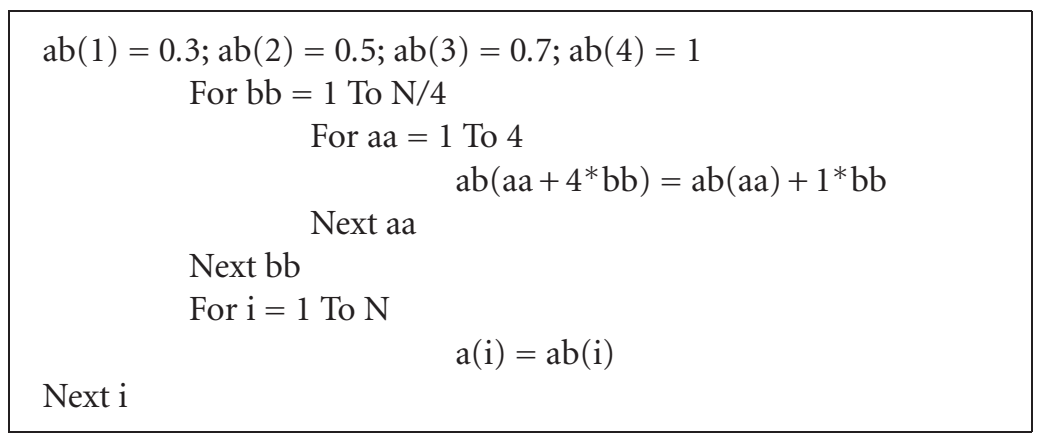

Algorithm 5.1. Arrival sequence for hybrid systems.

TABLE 5.1. Statistical analyses of various methods at the 500th generation.

\begin{tabular}{cccccccc}
\hline $\begin{array}{c}\text { Method } \\
\text { number }\end{array}$ & Method & Average & Best & Worst & SD & CV & Avedev \\
\hline 1 & WA & 15379.9102 & 13907.6580 & 19786.4941 & 170.9924 & 0.0111 & 62.1193 \\
2 & WB & 7891.9087 & 7795.7406 & 8190.4794 & 25.5744 & 0.0032 & 14.0143 \\
3 & WC & 7901.4545 & 7777.6306 & 8292.2425 & 20.4858 & 0.0026 & 10.1641 \\
4 & XA & 17376.4738 & 14081.6692 & 20189.4607 & 180.6685 & 0.0104 & 91.4140 \\
5 & XB & 9004.9407 & 8055.2561 & 10690.0855 & 24.8136 & 0.0028 & 37.1037 \\
6 & XC & 9398.6967 & 8174.7296 & 11432.7873 & 62.7363 & 0.0067 & 32.0660 \\
7 & YA & 17221.8462 & 14097.6442 & 19386.2370 & 177.1593 & 0.0103 & 131.8242 \\
8 & YB & 7815.1086 & 7769.5156 & 7933.5245 & 2.4994 & 0.0003 & 1.2602 \\
9 & YC & 7817.5676 & 7763.1663 & 7895.4521 & 3.5350 & 0.0005 & 1.9573 \\
10 & ZA & 15750.0587 & 12465.2807 & 17613.1775 & 58.2452 & 0.0037 & 26.0689 \\
11 & ZB & 7858.3669 & 7795.8160 & 8026.0831 & 6.9454 & 0.0009 & 3.4780 \\
12 & ZC & 7861.0846 & 7779.7099 & 8011.0604 & 14.6579 & 0.0019 & 9.4978 \\
\hline
\end{tabular}

(iii) Number of jobs $=50$, and

(iv) Total number of run (simulation) $=1000$.

The arrival sequence ( $a_{i}$ for $i=1$ to $N$ ) for $N=50$ is obtained from Algorithm 5.1.

The PSO algorithms associated with the 12 methods are simulated 1000 times at different intervals of time. The optimal control variable $\left(u_{i}\right)$, the departure time $\left(x_{i}\right.$ for $i=1,2, \ldots, 50)$ and the fitness objective function $(J)$ are computed for all 12 methods. The average value of the optimal control variable $\left(u_{i}\right)$, and the corresponding departure time $\left(x_{i}\right)$ are presented in Tables 5.5 and 5.6. The cost or fitness value of the objective function which represents the class of single-stage hybrid system is recorded for every 500 generations and their statistical analyses are compared in Tables 5.1-5.4.

All the 12 methods are executed through visual basic program and the fitness values for all the methods are taken by running the simulation 1000 times at different times. 
TABLE 5.2. Statistical analyses of various methods at the 1000th generation.

\begin{tabular}{cccccccc}
\hline $\begin{array}{c}\text { Method } \\
\text { number }\end{array}$ & Method & Average & Best & Worst & SD & CV & Avedev \\
\hline 1 & WA & 11983.6000 & 10168.3405 & 13881.3154 & 170.9860 & 0.0143 & 122.9995 \\
2 & WB & 7814.2846 & 7755.6382 & 8033.7556 & 20.1515 & 0.0026 & 11.3079 \\
3 & WC & 7820.6383 & 7764.4469 & 8097.1594 & 12.0986 & 0.0015 & 2.8512 \\
4 & XA & 13441.9106 & 10168.3405 & 16386.9300 & 78.3690 & 0.0058 & 46.8587 \\
5 & XB & 7921.0846 & 7772.2090 & 9475.1826 & 70.5600 & 0.0089 & 10.7831 \\
6 & XC & 8083.1708 & 7781.7568 & 9239.8054 & 63.8962 & 0.0079 & 30.9782 \\
7 & YA & 12383.4070 & 10567.3418 & 13685.8476 & 74.8552 & 0.0060 & 44.3305 \\
8 & YB & 7765.2765 & 7744.6986 & 7806.4233 & 1.8247 & 0.0002 & 1.2557 \\
9 & YC & 7768.6854 & 7742.2869 & 7801.9017 & 3.0599 & 0.0004 & 1.8542 \\
10 & ZA & 11172.4901 & 9643.2855 & 13158.6611 & 108.8048 & 0.0097 & 104.0414 \\
11 & ZB & 7778.0332 & 7744.3391 & 7825.2758 & 7.2364 & 0.0009 & 1.6913 \\
12 & ZC & 7778.1319 & 7744.8737 & 7837.4551 & 2.7583 & 0.0004 & 1.8686 \\
\hline
\end{tabular}

TABLE 5.3. Statistical analyses of various methods at the 1500th generation.

\begin{tabular}{cccccccc}
\hline $\begin{array}{c}\text { Method } \\
\text { number }\end{array}$ & Method & Average & Best & Worst & SD & CV & Avedev \\
\hline 1 & WA & 8608.4865 & 8091.6661 & 9669.7744 & 45.5096 & 0.0053 & 27.0239 \\
2 & WB & 7789.7420 & 7748.8893 & 7862.9554 & 5.4448 & 0.0007 & 3.5318 \\
3 & WC & 7797.7273 & 7749.4070 & 7884.1366 & 3.7937 & 0.0005 & 1.9491 \\
4 & XA & 10545.2723 & 8091.6661 & 13129.3045 & 99.6716 & 0.0095 & 50.2086 \\
5 & XB & 7778.7645 & 7744.7194 & 7988.4480 & 11.3561 & 0.0015 & 2.4609 \\
6 & XC & 7792.4137 & 7748.8792 & 8085.4559 & 19.0309 & 0.0024 & 7.5050 \\
7 & YA & 8749.2009 & 8201.6420 & 9651.5879 & 58.3183 & 0.0067 & 38.0115 \\
8 & YB & 7753.3627 & 7740.4907 & 7782.9877 & 1.7317 & 0.0002 & 0.8079 \\
9 & YC & 7754.8549 & 7738.6329 & 7774.2684 & 1.7599 & 0.0002 & 1.1162 \\
10 & ZA & 8859.8511 & 8170.7338 & 9804.0423 & 14.0901 & 0.0016 & 15.3803 \\
11 & ZB & 7759.7919 & 7739.6439 & 7792.9689 & 0.7206 & 0.0001 & 0.7065 \\
12 & ZC & 7759.8744 & 7740.0971 & 7783.7389 & 1.6642 & 0.0002 & 0.7627 \\
\hline
\end{tabular}

The average values (Mean), best and worst fitness values among 1000 simulated results and the standard deviations (SD) of the fitness values for each method are calculated. In order to strengthen the comparison, few more statistics tests are conducted, the coefficient variance, which is calculated from the ratio of standard deviation to the mean and the average deviation, which will, give the average of the absolute deviation of the 
TABLE 5.4. Statistical analyses of various methods at the 2000th generation.

\begin{tabular}{cccccccc}
\hline $\begin{array}{c}\text { Method } \\
\text { number }\end{array}$ & Method & Average & Best & Worst & SD & CV & Avedev \\
\hline 1 & WA & 7846.1401 & 7746.8580 & 8271.0692 & 18.9162 & 0.0024 & 6.4859 \\
2 & WB & 7778.5150 & 7745.7858 & 7851.7101 & 3.2332 & 0.0004 & 2.1306 \\
3 & WC & 7785.0885 & 7743.6033 & 7878.1134 & 3.2447 & 0.0004 & 0.8094 \\
4 & XA & 8775.0653 & 7760.2905 & 9929.4221 & 185.4796 & 0.0211 & 100.8503 \\
5 & XB & 7756.7710 & 7737.9135 & 7789.3040 & 1.9710 & 0.0003 & 0.9622 \\
6 & XC & 7760.4903 & 7739.8483 & 7892.4369 & 7.9065 & 0.0010 & 1.8714 \\
7 & YA & 7814.6565 & 7763.5000 & 7937.2679 & 8.9888 & 0.0012 & 5.1735 \\
8 & YB & 7747.9780 & 7734.8516 & 7765.4052 & 0.7423 & 0.0001 & 0.3795 \\
9 & YC & 7749.1308 & 7736.5328 & 7766.8408 & 1.6399 & 0.0002 & 1.0236 \\
10 & ZA & 7935.2318 & 7802.2804 & 8476.1164 & 20.9959 & 0.0026 & 4.8424 \\
11 & ZB & 7750.9974 & 7735.9609 & 7770.2229 & 0.3444 & 0.0000 & 0.2394 \\
12 & ZC & 7751.6891 & 7738.2488 & 7771.5835 & 1.3291 & 0.0002 & 0.5233 \\
\hline
\end{tabular}

fitness values from their mean, which are taken in 1000 simulation runs. Added to these analyses, hypothesis t-test: analysis of variance (ANOVA) also carried out to validate the efficacy among the proposed algorithms. These statistics analysis are presented in Tables 5.1-5.4. The graphical analysis is done through Box plot, which is shown in Figure 5.3.

In order to ease the analysis, all the 12 methods are compared with respect to group 1 and group 2 classification. That is, all the methods in group 1 are compared with each of the inertia weight given in group 2 and vice versa. The comparisons of fitness values between each method are done in 3 dimensional plots using MATLAB. And they are shown in Figures 5.1 and 5.2.

The optimal control variables $\left(u_{1}, u_{2}, \ldots, u_{50}\right)$ in $(5.2)$ and the departure time of each job $\left(x_{1}, x_{2}, \ldots, x_{50}\right)$ in $(5.3)$ are computed for all 12 methods and tabulated in Tables 5.5 and 5.6, respectively. From the departure time of each job, the queue lengths of the server (of the single-stage hybrid system) at the arrival times $\left(a_{1}, a_{2}, \ldots, a_{50}\right)$ are computed and plotted in Figure 5.4 for all the 12 methods individually.

The dynamic behavior of each particle in the search space for each method with $N=$ 50 is taken over 2000 generations and the particles positions are recorded and presented in Table 5.7 and Figure 5.5. The particle positions for the methods which are with $\mathrm{W}$ and A are moving away form the equilibrium point (the position where the optimal solution is obtained) often and takes a lot of generations to settle whereas in methods comprising of $\mathrm{Y}, \mathrm{Z}$ and $\mathrm{B}$ (sometimes $\mathrm{C}$ ), the particle positions are always in a closer range of the equilibrium point and converge faster.

The execution times for each method are calculated for every simulation and hence the average execution time is calculated and presented in Table 5.8 and Figure 5.6, from which it is understood that methods comprises of $\mathrm{Y}$ and $\mathrm{Z}$ are yield the optimal solution faster with less execution time. 
268 Optimal control of hybrid systems via new PSO algorithm

TABLE 5.5. Comparison of optimal control variable $\left(u_{i}\right)$ for all 12 methods.

\begin{tabular}{|c|c|c|c|c|c|c|c|c|c|c|c|c|c|}
\hline \multirow{2}{*}{\multicolumn{2}{|c|}{$\begin{array}{c}\text { Arrival } \\
\text { a(i) }\end{array}$}} & \multicolumn{3}{|c|}{ W } & \multicolumn{3}{|c|}{$\mathrm{X}$} & & \\
\hline & & A & B & C & A & B & C & A & B & C & A & B & C \\
\hline 1 & 0.3 & 241 & .233 & 213 & 1 & 0.259 & 230 & 0.221 & 0.249 & .211 & & 232 & \\
\hline 2 & & & 246 & & & & & & & & & & \\
\hline 3 & 0 & & 82 & & & & & & & & & & \\
\hline 4 & 1 & & 277 & & & & & & & & & & \\
\hline 5 & 1.3 & & 177 & & & 4 & & & & & & & \\
\hline 6 & & & 246 & & & 11 & & & & & & & \\
\hline 7 & 1 & & 260 & & & 273 & & & & & & & \\
\hline 8 & 2 & & 272 & & & 295 & & & & & & & \\
\hline 9 & 2. & & 221 & & & & & & & & & & \\
\hline 10 & & & 224 & & & & & & & & & & \\
\hline 11 & & & 281 & & & & & & & & & & 07 \\
\hline 12 & 3 & & 79 & & & & & & & & & & \\
\hline 13 & & & & & & & & & & & & & \\
\hline 4 & & & & & & & & & & & & & \\
\hline 15 & & & & & & & & & & & & & \\
\hline 16 & 4 & & & & & & & & & & & & \\
\hline 17 & & & & & & & & & & & & & \\
\hline 18 & & & 3 & & & & & & & & & & \\
\hline 19 & 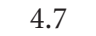 & & 1 & & & & & & & & & & \\
\hline 20 & 5 & & & & & & & & & & & & \\
\hline 21 & & & & & & & & & & & & & \\
\hline 22 & & & & & & & & & & & & & \\
\hline 23 & 5. & & & & & & & & & & & & \\
\hline 24 & 6 & & & & & & & & & & & & \\
\hline 25 & 6.3 & & & & & & & & & & & & \\
\hline 26 & & & 4 & & & & & & & & & & \\
\hline 27 & 6 & & & & & & & & & & & & \\
\hline 28 & 7 & & & & & & & & & & & & \\
\hline 29 & & & & & & & & & & & & & \\
\hline 30 & & & & & & & & & & & & & \\
\hline 31 & 7 & & & & & & & & & & & & \\
\hline 32 & 8 & & & & & & & & & & & & 51 \\
\hline 33 & & & & & & & & & & & & & \\
\hline 34 & & & & & & & & & & & & & \\
\hline 35 & & & & & & & & & & & & & 75 \\
\hline 36 & 9 & & & & & & & & & & & & \\
\hline 37 & & & 1 & & & & & & & & & & 0.227 \\
\hline 38 & & & & & & & & & & & & & 34 \\
\hline 20 & & & & & & & & & & & & & \\
\hline 40 & & & & & & & & & & & & & 95 \\
\hline 41 & & & & & & & & & & & & & \\
\hline 42 & & & & & & & & & & & & & 0.271 \\
\hline 43 & 10 & & & & & & & & & & & & 0.290 \\
\hline 44 & 11 & & & & & & & & & & & & \\
\hline 45 & 11 & & & & & & & & & & & & \\
\hline 46 & 11 & & & & & & & & & & & & \\
\hline 47 & 11 & & & & & & & & & & & & \\
\hline 48 & 12 & & & & & & & & & & & & \\
\hline 49 & 12 & & & & & & & & & & & & \\
\hline 50 & 12. & & 929 & 741 & 0.900 & 0.770 & 0.773 & 0.733 & 0.819 & 0.795 & 0.597 & 0.819 & 0.795 \\
\hline
\end{tabular}


TABle 5.6. Comparison of departure time $\left(x_{i}\right)$ of each job.

\begin{tabular}{|c|c|c|c|c|c|c|c|c|c|c|c|c|c|}
\hline \multirow{2}{*}{\multicolumn{2}{|c|}{$\begin{array}{c}\text { Arrival } \\
\text { a(i) }\end{array}$}} & \multicolumn{3}{|c|}{$\overline{\mathrm{W}}$} & \multicolumn{3}{|c|}{ X } & & \\
\hline & & A & B & C & A & B & C & A & B & C & 11 & B & C \\
\hline 1 & 0.3 & 0.541 & 0.533 & 513 & 534 & 0.559 & 0.530 & 521 & 0.549 & 511 & 571 & 532 & \\
\hline 2 & 0.5 & & 779 & & & & & & & & & 748 & \\
\hline 3 & 0.7 & & 1.061 & & & 48 & & & & & & 005 & \\
\hline 4 & 1 & & 1.338 & & 268 & & & & 1.300 & & & 300 & \\
\hline 5 & 1.3 & & 1.515 & & & 1.506 & & & & & & 558 & \\
\hline 6 & 1.5 & 836 & 1.761 & & 754 & 1.747 & & & 1.798 & & & 801 & \\
\hline 7 & 1.7 & 053 & 2.022 & & & 2.020 & & & 2.067 & & & 066 & \\
\hline 8 & 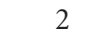 & 304 & 2.293 & & 2397 & 2.315 & & & 2.301 & & & 313 & \\
\hline 9 & 2.3 & 56 & 2.521 & 15 & 2621 & 2.601 & & & & & & 569 & \\
\hline & 2.5 & 781 & 2.745 & & & 831 & & & & & & 783 & \\
\hline & 2.7 & & 3.026 & & & 066 & & & & & & 082 & \\
\hline & 3 & & .305 & & & 00 & & & & & & 300 & \\
\hline & 3.3 & & 35 & & & 511 & & & & & & 556 & \\
\hline & 3 & & 3.769 & & & 771 & & & & & & 764 & \\
\hline & 3.7 & & 999 & & & & & & & & & 83 & \\
\hline & 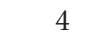 & & 301 & & & & & & & & & 300 & \\
\hline & 4.3 & & 4.568 & & & & & & & & & 543 & \\
\hline & 4 & & 4.811 & & & & & & & & & 768 & \\
\hline & 4.7 & & 5.072 & & & & & & & & & 15 & \\
\hline & 5 & & 5.302 & & & & & & & & & 300 & \\
\hline & 5 & & 5.506 & & & & & & & & & 28 & \\
\hline & & & 5.718 & & & & & & & & & & \\
\hline & 5.7 & 4 & 6.052 & & & & & & & & & 31 & \\
\hline & 6 & & 6.297 & & & & & & & & & 300 & \\
\hline & 6.3 & & 6.585 & & & & & & & & & 576 & \\
\hline & 6 & 822 & 6.859 & & & & & & & & & 96 & \\
\hline & 6.7 & & .089 & & & & & & & & & 56 & \\
\hline & 7 & & 299 & & & & & & & & & 300 & \\
\hline & 7 & & 525 & & & & & & & & & & \\
\hline & 7 & & 7.781 & & & & & & & & & 57 & \\
\hline & 7.7 & & .039 & & & & & & & & & 53 & \\
\hline & 8 & & 02 & & & & & & & & & 00 & \\
\hline 3 & 8.3 & & 03 & & & & & & & & & 532 & \\
\hline & 8. & & & & & & & & & & & 69 & \\
\hline & 8.7 & & & & & & & & & & & 20 & 20 \\
\hline & 9 & & & & & & & & & & & 00 & \\
\hline & 9. & & & & & & & & & & & 21 & \\
\hline & 9 & & 9.767 & & 10. & 9.751 & & 9.7 & 9.775 & 9.768 & 9.769 & 9.756 & 61 \\
\hline & 9. & & & & & & & & & & & 00 & \\
\hline & & & & & & & & & & & & & \\
\hline & & & & & & & & & & & & & \\
\hline & & & & & & & & & & & & & \\
\hline & & & & & & & & & & & & & \\
\hline & 11 & & & & & & & & & & & & \\
\hline & & & & & & & & & & & & & \\
\hline & & & & & & & & & & & & & \\
\hline & 11 & & & & & & & & & & & & \\
\hline & 12 & & & & & & & & & & & & \\
\hline & 12 & & & & & & & & & & & & \\
\hline & & & & & & & & & & & & & \\
\hline
\end{tabular}


270 Optimal control of hybrid systems via new PSO algorithm

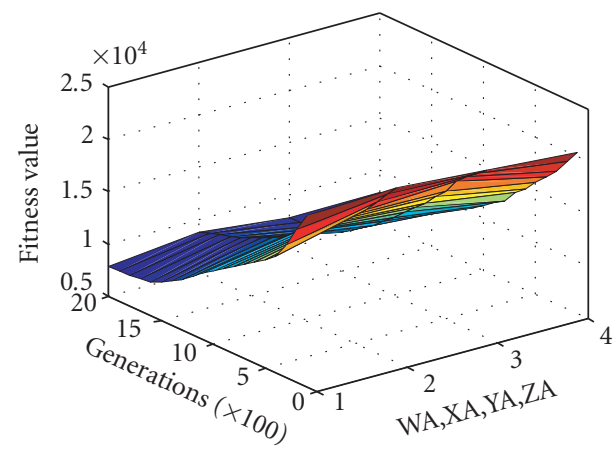

(a)

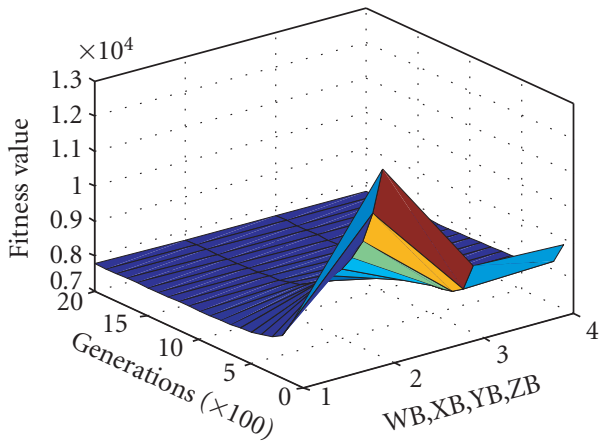

(b)

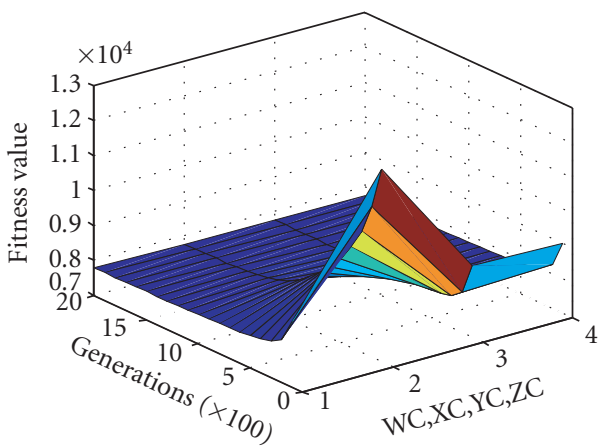

(c)

FIgURE 5.1. Three-dimensional plot of the comparison between methods. (a) WA, XA, YA, and ZA; (b) WB, XB, YB, and ZB; (c) WC, XC, YC, and ZC. 


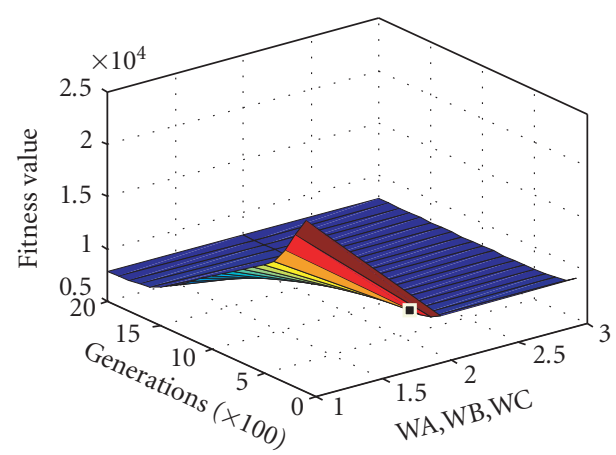

(a)

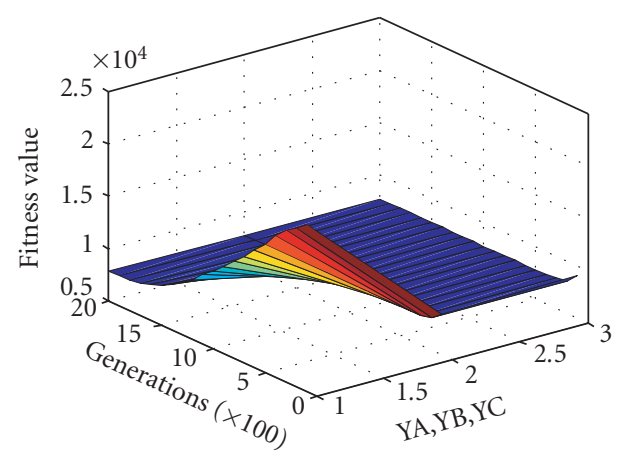

(c)

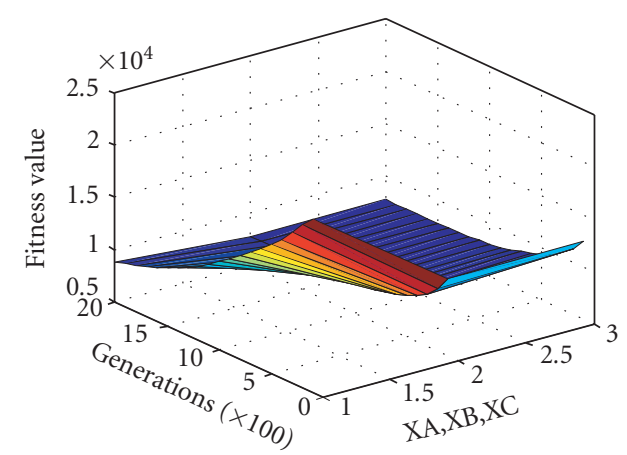

(b)

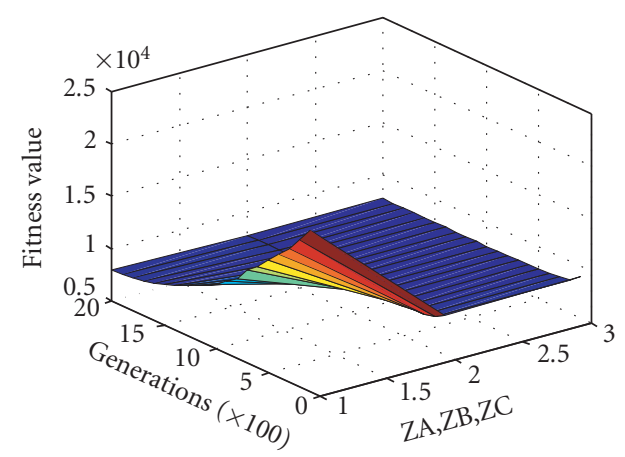

(d)

FigURE 5.2. Three-dimensional plot of the comparison between methods. (a) WA, WB, and WC; (b) $\mathrm{XA}, \mathrm{XB}$, and XC; (c) YA, YB, and YC; (d) ZA, ZB, and ZC.

\section{Discussions and conclusion}

In this paper, new variants to the inertia weight and velocity equations are considered and they are classified into 12 methods. In order to compare the validity and usefulness of the proposed velocity equations in PSO methods with the existing standard PSO (method WA), all the methods are simulated 1000 times at different periods of time, and 1000 simulated results for each method are taken at different timings. The performance of different algorithms is compared with respect to the solution accuracy in the fitness, the standard deviations, co-efficient variance, average deviation, ANOVA t-test, and the percentage of deviation in the fitness from the proposed best method.

From the results stated in Table 5.1-5.4, it is obvious that the method $8(Y B)$ is the best followed by method 9 (YC). This clearly establishes the fact that method Y with $\mathrm{B}$ and $\mathrm{C}$ yields better solutions. This is the most significant outcome of the experiments 


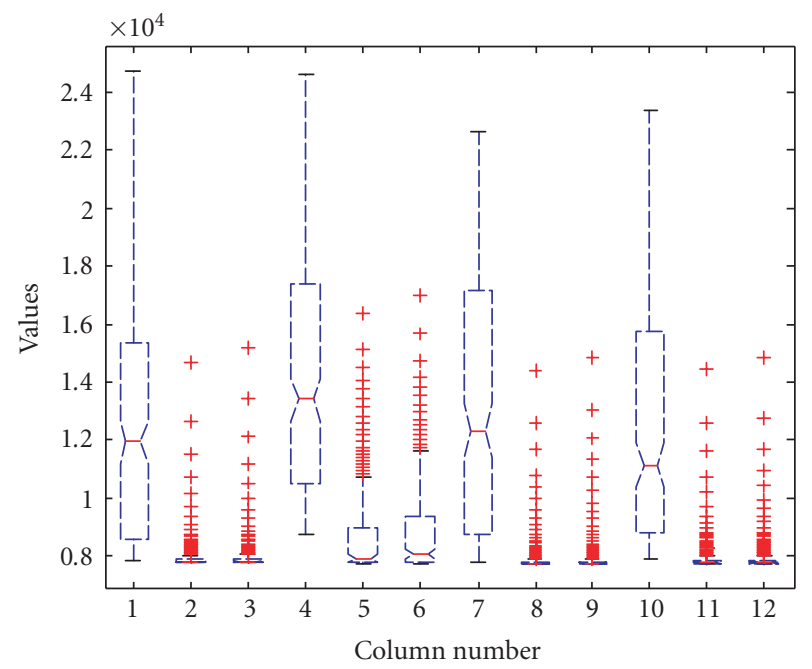

Figure 5.3. ANOVA t-test analysis of fitness values over 2000 generations for all 12 methods.

performed. These combinations have been shown to work efficiently with regard to an optimal control problem here but it is believed that these might be equally efficient with regard to all other problems where PSO can be used. All the 12 methods are sorted on the average fitness value, and their rankings are as follows: $\mathrm{YB}, \mathrm{YC}, \mathrm{ZB}, \mathrm{ZC}, \mathrm{XB}, \mathrm{XC}, \mathrm{WB}$, WC, YA, WA, ZA, and XA. From the above rankings, it is very obvious that the methods comprise of $\mathrm{Y}$ and $\mathrm{B}$ always provide better results compared to other methods. It is also observed that except methods ZA and XA all other methods are better than the standard $\mathrm{PSO}$, which is known here as method WA.

By analyzing the PSO parameters, it is observed that normally the cognitive $\left(c_{1}\right)$ and social $\left(c_{2}\right)$ parameters are set to 2 for a better convergence. This is proved in the proposed methods. In method X, these two constants $\left(c_{1}\right.$ and $c_{2}$ ) are always greater than or equal to 2 whereas they $\left(c_{1}\right.$ and $\left.c_{2}\right)$ are always less than or equal to 2 in method $Z$, since pbest ${ }_{i} \geq$ gbest, for $i=1,2, \ldots, 50$. In method $\mathrm{Y}$, they $\left(c_{1}\right.$ and $\left.c_{2}\right)$ are always equal to 2 . PSO algorithm with method $\mathrm{Y}$ always yields better result followed by those comprise of method $\mathrm{Z}$ with respect to the optimal fitness solution. It is also observed from the Table 5.1-5.4, method $\mathrm{Z}$ is always yielding the optimal solution with less standard deviation, which proves this method's accuracy and consistency. So from consistency point of view, PSO algorithm with method $\mathrm{Z}$ is a better choice than the one with method $\mathrm{Y}$.

From the hypothesis ANOVA t-test that is shown in Figure 5.3, it can be easily concluded that method YB (refers to method 8 in the figure) is better than any other methods considered in this paper. This box plot representation provides an excellent visual summary of many important aspects of a distribution of fitness value over 2000 generations. The graphical view clearly shows the effectiveness of the proposed algorithms and their fitness distribution. 

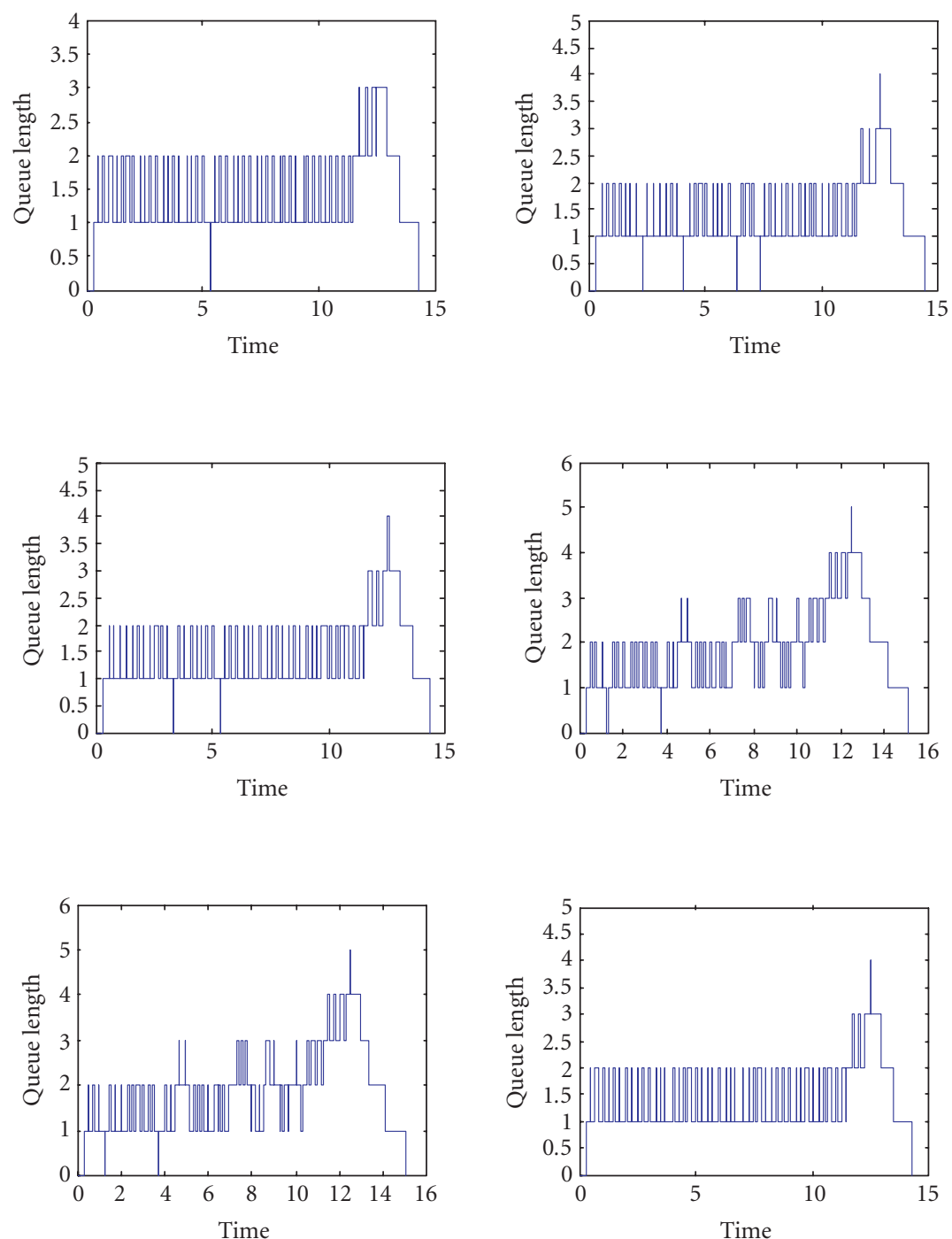

FIGURE 5.4. Queue length versus arrival time for methods WA, WB, WC, XA, XB, XC, YA, YB, YC, $\mathrm{ZA}, \mathrm{ZB}$, and $\mathrm{ZC}$, respectively.

Figure 5.4 provides the information about the queue length in the server at the arrival time of each job. The queue lengths obtained through methods YB, YC, ZB, and ZC are more or less the same and proves their superiority among the other methods.

Figure 5.5 presents the dynamic behavior of each particle in the search space for each method, which is taken over 2000 generations. The particle positions for the methods with $\mathrm{W}$ and $\mathrm{A}$ are moving away form the equilibrium point (the position where the 
274 Optimal control of hybrid systems via new PSO algorithm
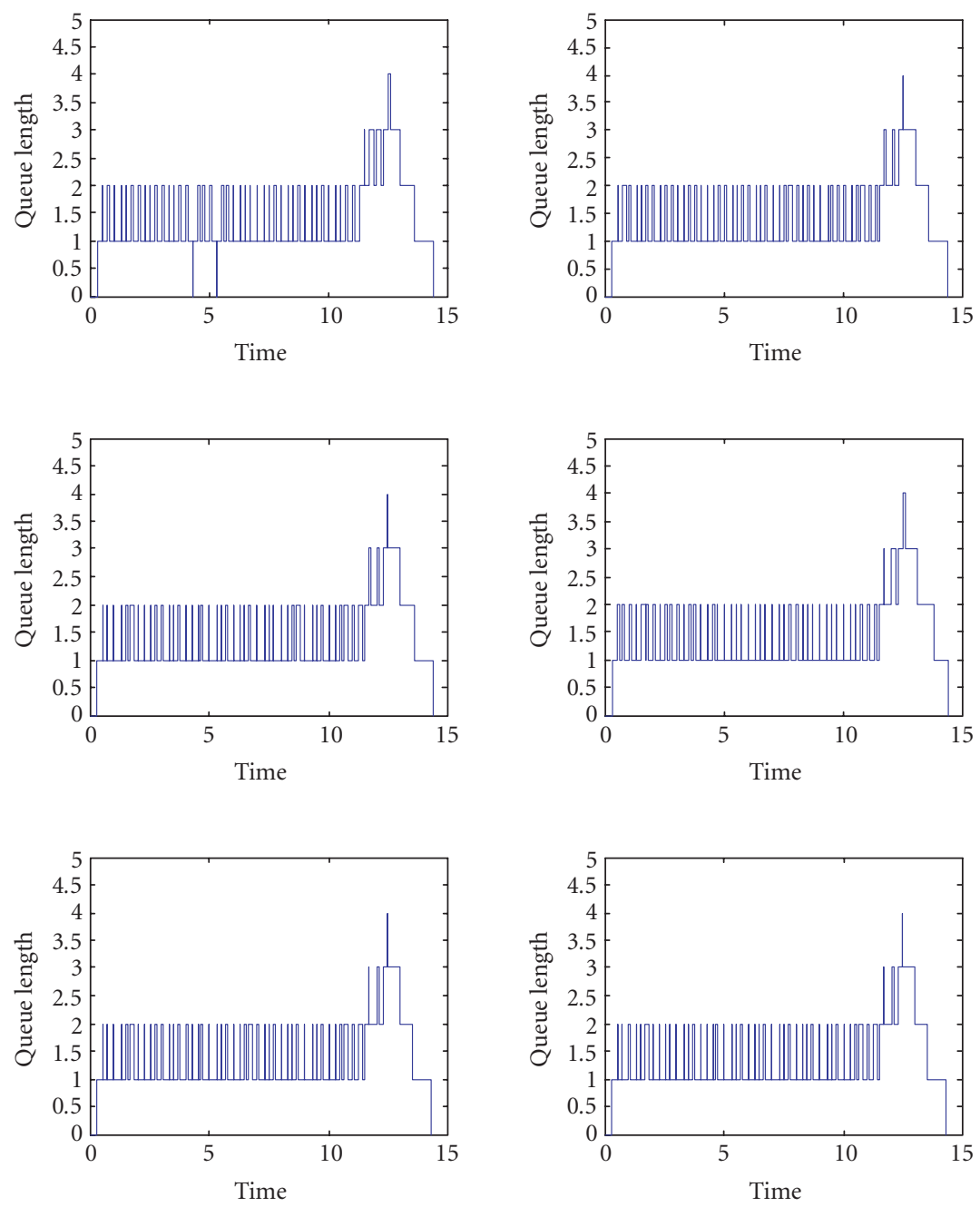

Figure 5.4. Continued.

optimal solution is obtained) often and takes a lot of generations to settle whereas in methods with Y, Z, and B (sometimes C), the particle positions are always in a closer range of the equilibrium point and converge faster. It is very obvious that the particle positions in methods $\mathrm{YB}, \mathrm{YC}, \mathrm{ZB}$, and $\mathrm{ZC}$ are in a very narrow range, which implies how fast they are reaching the equilibrium point to obtain the optimal solution. This again proves the effectiveness and faster convergence capability of the methods $\mathrm{YB}, \mathrm{YC}, \mathrm{ZB}$, and ZC.

The most important aspect of simulation program is its execution time. Any algorithm, which runs at less execution time compared to other algorithms, is considered as best method. From Table 5.8 and Figure 5.6, it is clearly identified that among the 12 


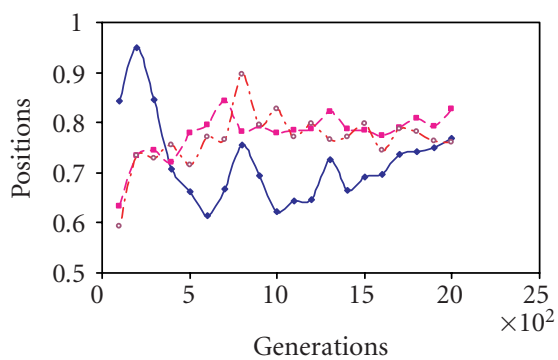

$$
\begin{aligned}
& -\square \text { WA } \\
& -- \text { WB } \\
& -\cdots \text { WC }
\end{aligned}
$$

(a)

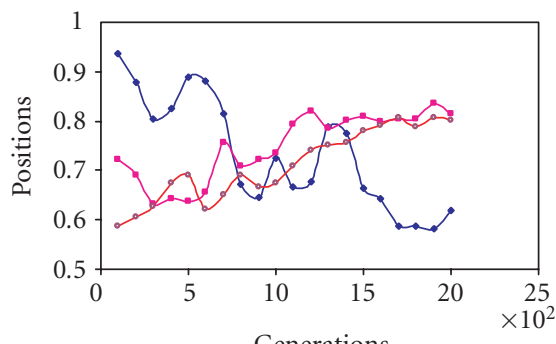

Generations

$$
\begin{array}{r}
\longrightarrow \mathrm{XA} \\
\because \mathrm{XB}
\end{array}
$$

(b)

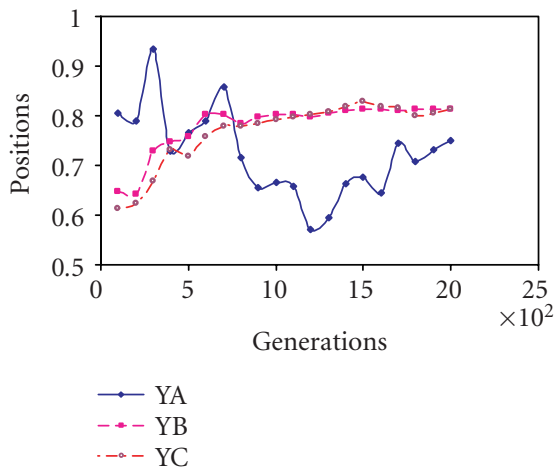

(c)

Figure 5.5. Comparison of the particle's best position for methods. (a) WA, WB, and WC; (b) XA, $\mathrm{XB}$, and $\mathrm{XC}$; (c) YA, YB, and YC; (d) ZA, ZB, and ZC; (e) WA, XA, YA, and ZA; (f) WB, XB, YB, and $\mathrm{ZB}$; (g) WC, XC, YC, and ZC. 
276 Optimal control of hybrid systems via new PSO algorithm

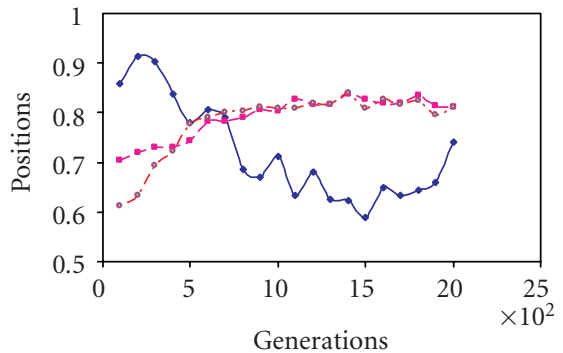

$$
\begin{aligned}
& \cdots \mathrm{ZA} \\
& \cdots-\mathrm{ZB} \\
& \cdots-\mathrm{ZC}
\end{aligned}
$$

(d)

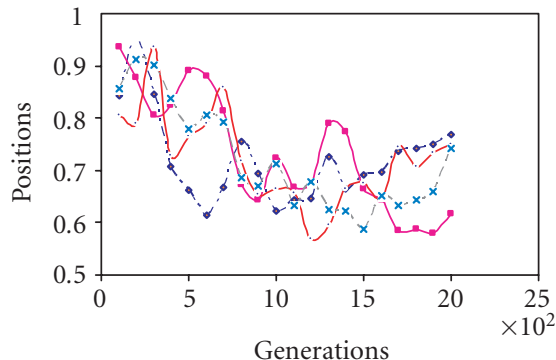

$$
\begin{array}{ll}
\cdots \text { WA } & -- \text { YA } \\
\rightarrow \text { XA } & --x-\text { ZA }
\end{array}
$$

(e)

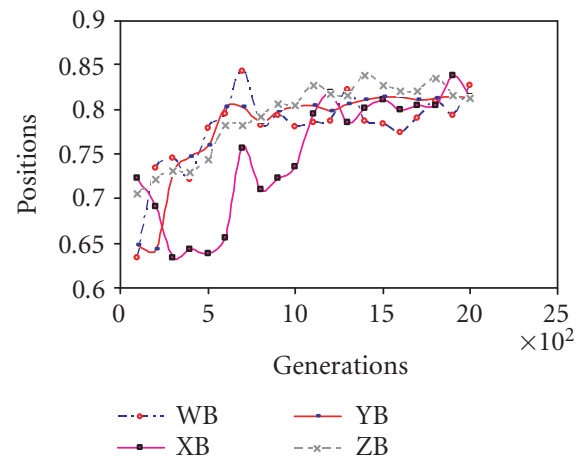

(f)

Figure 5.5. Continued. 


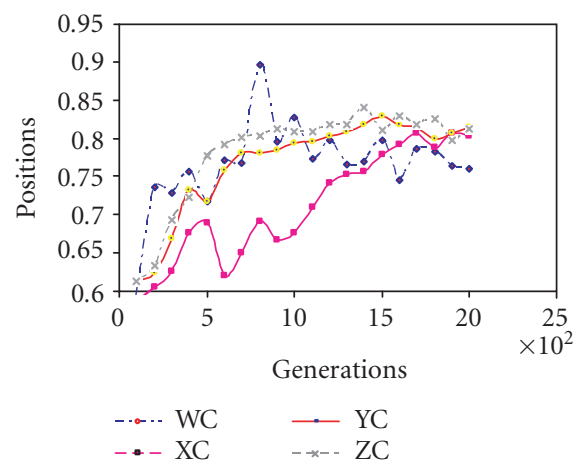

$(\mathrm{g})$

Figure 5.5. Continued.

TABLE 5.7. Dynamic behavior of particles best position in each generation.

\begin{tabular}{|c|c|c|c|c|c|c|c|c|c|c|c|c|}
\hline \multirow{2}{*}{$\begin{array}{l}\text { Gener } \\
\text { ation }\end{array}$} & \multicolumn{3}{|c|}{ W } & \multicolumn{3}{|c|}{$\mathrm{X}$} & \multicolumn{3}{|c|}{$\mathrm{Y}$} & \multicolumn{3}{|c|}{ Z } \\
\hline & A & B & C & 4 & B & C & A & B & C & A & B & 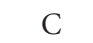 \\
\hline 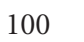 & 0.8436 & & & & & & & & & & & \\
\hline & & & & & & & & & & & & \\
\hline & & & & & & & & & & & & \\
\hline & & & & & & & & & & & & \\
\hline 00 & & & & 9 & & & & & & & & 0 \\
\hline & & & & & & & & & & & & c \\
\hline 800 & & & & & & & & & & & & c \\
\hline & & & & & & & & & & & & 0 \\
\hline & & & & & & & & & & & & \\
\hline (n) & & & & & & & & & & & & 0.808 \\
\hline 1100 & & & & & & 0 & & & & & & 307 \\
\hline (200 & & & & & & & & & & & & 0 \\
\hline 300 & & & & & & & & & & & & 0.8173 \\
\hline & & & & & & & & & & & & 0 \\
\hline & & & & & & & & & & & & 309 \\
\hline 00 & 0 & 0. & 0. & 0. & 0. & 0 & & 0. & & & & 0.8284 \\
\hline 1700 & 0.7356 & 0 . & 0 & 0 & 0 . & 0 & 4 & 0 . & 0 & 8 & 9 & 0.8171 \\
\hline (c) & & & & & 0. & 0 & 0 & 0. & 0 & & & 0.825 \\
\hline 1900 & 91 & 0.7 & 36 & 309 & 0.8 & 0.8066 & 27 & 0.8130 & 0.8066 & 1 & 154 & 0.7967 \\
\hline ( & & & & 0 & 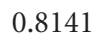 & 0 & ${ }^{0}$ & 0.8127 & 0.01 & 0.1 & 0.0121 & 0.012 \\
\hline
\end{tabular}


TABLE 5.8. Execution time for simulation of various PSO methods.

\begin{tabular}{ccccccccccccc}
\hline Method & WA & WB & WC & XA & XB & XC & YA & YB & YC & ZA & ZB & ZC \\
\hline $\begin{array}{c}\text { Execution time } \\
\text { (in seconds) }\end{array}$ & 7.35 & 7.3 & 7.33 & 6.89 & 6.74 & 6.77 & 6.52 & 6.37 & 6.4 & 6.71 & 6.58 & 6.67 \\
\hline
\end{tabular}

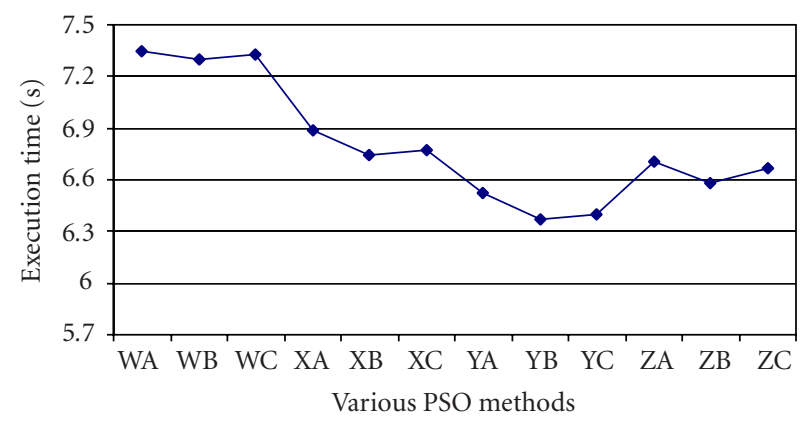

FIGURE 5.6. The average execution time of various PSO methods.

methods considered, three methods those comprise of method Y are having less execution time. This proves the superior capability of method Y. Algorithms with method Z are in the second rank, which are better than the methods X and W.

In summary, it is proven in so many aspects that the proposed methods, those with $\mathrm{Y}$ and also $\mathrm{Z}$ are much better than the existing methods and other proposed methods. Hence it can be concluded that the PSO algorithms with method $\mathrm{Y}$ is the superior among others and in particular it is more effective with the inertia weight which given in method $\mathrm{B}$ in computing the optimal control and fitness solution of the single-stage hybrid system.

\section{References}

[1] C. G. Cassandras, D. L. Pepyne, and Y. Wardi, Optimal control of a class of hybrid systems, IEEE Trans. Automat. Control 46 (2001), no. 3, 398-415.

[2] M. Clerc and J. Kennedy, The particle swarm: Explosion, stability, and convergence in a multidimensional complex space, IEEE Trans. Evol. Comput. 6 (2002), no. 1, 58-73.

[3] J. Kennedy, The particle swarm: social adaptation of knowledge, Proc. IEEE International Conference on Evolutionary Computation (Indianapolis, Indiana), IEEE Service Center, New Jersey, 1997, pp. 303-308.

[4] J. Kennedy and R. C. Eberhart, Particle swarm optimization, Proc. IEEE International Conference on Neural Networks (Perth, Australia), IEEE Service Center, New Jersey, 1995, pp. 1942-1948.

[5] S. Naka, T. Genji, T. Yura, and Y. Fukuyama, A hybrid particle swarm optimization for distribution state estimation, IEEE Trans. on Power Systems 18 (2003), no. 1, 60-68.

[6] D. L. Pepyne and C. G. Cassandras, Modeling, analysis, and optimal control of a class of hybrid systems, Discrete Event Dyn. Syst. 8 (1998), no. 2, 175-201. 
[7] Y. Shi and R. C. Eberhart, Empirical study of particle swarm optimization, Proceedings of the Congress on Evolutionary Computation (Washington D.C., 1999), IEEE Service Center, New Jersey, 1999, pp. 1945-1950.

[8] P. Zhang and C. G. Cassandras, An improved forward algorithm for optimal control of a class of hybrid systems, IEEE Trans. Automat. Control 47 (2002), no. 10, 1735-1739.

M. Senthil Arumugam: Faculty of Engineering \& Technology (FET), Multimedia University (Melaka Campus), Jalan Ayer Keroh Lama, Bukit Beruang, 75450 Melaka, Malaysia

E-mail address: msenthil.arumugam@mmu.edu.my

M. V. C. Rao: Faculty of Engineering \& Technology, Multimedia University (Melaka Campus), Jalan Ayer Keroh Lama, Bukit Beruang, 75450 Melaka, Malaysia

E-mail address: machavaram.venkata@mmu.edu.my 


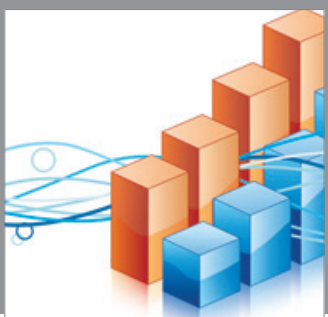

Advances in

Operations Research

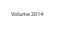

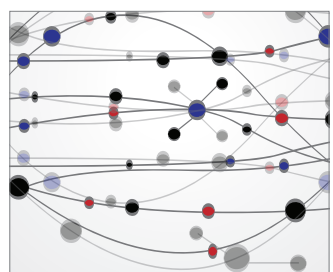

\section{The Scientific} World Journal
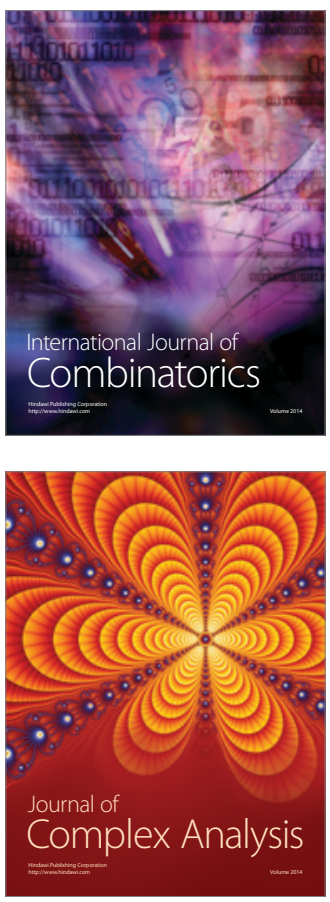

International Journal of

Mathematics and

Mathematical

Sciences
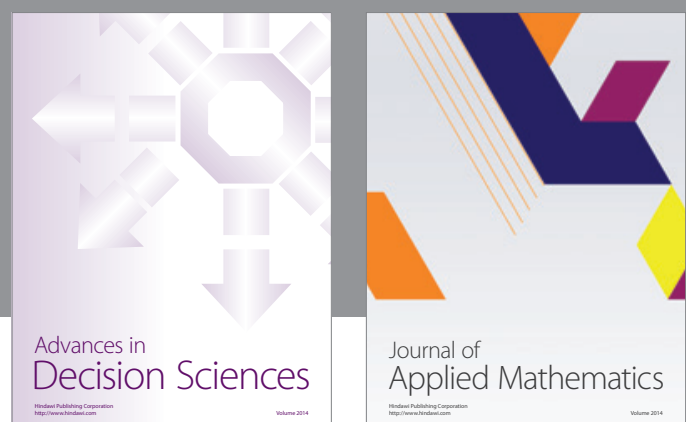

Journal of

Applied Mathematics
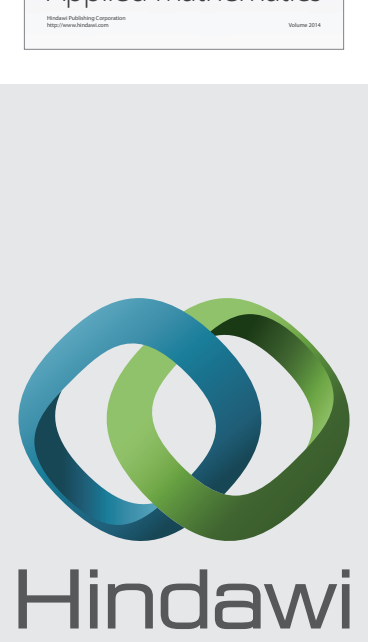

Submit your manuscripts at http://www.hindawi.com
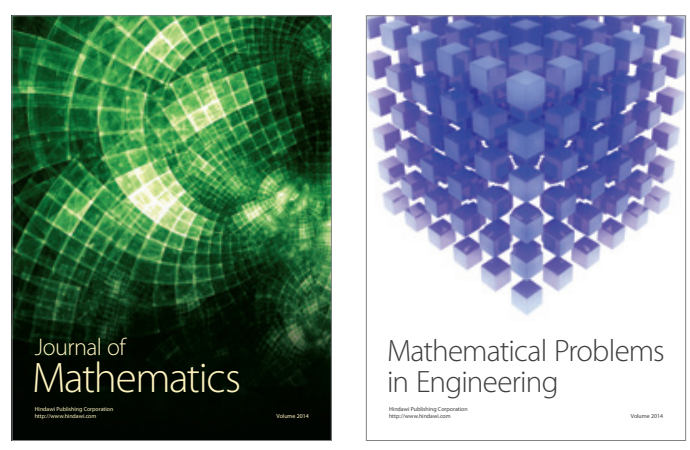

Mathematical Problems in Engineering
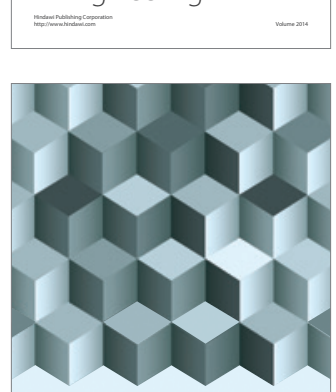

Journal of

Function Spaces
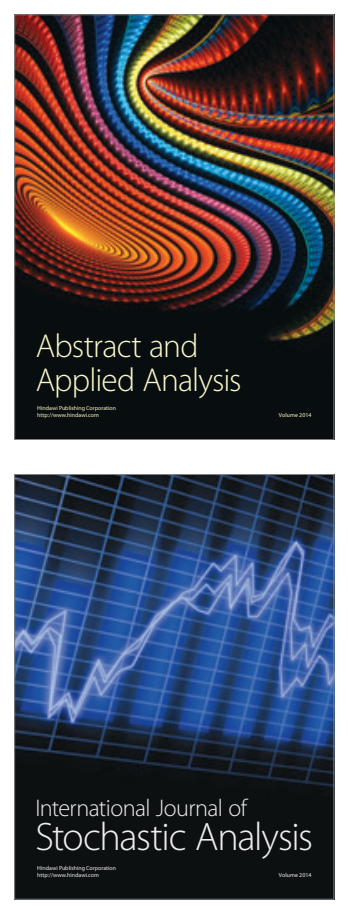

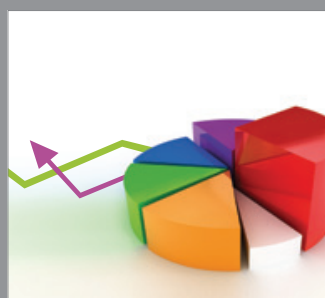

ournal of

Probability and Statistics

Promensencen
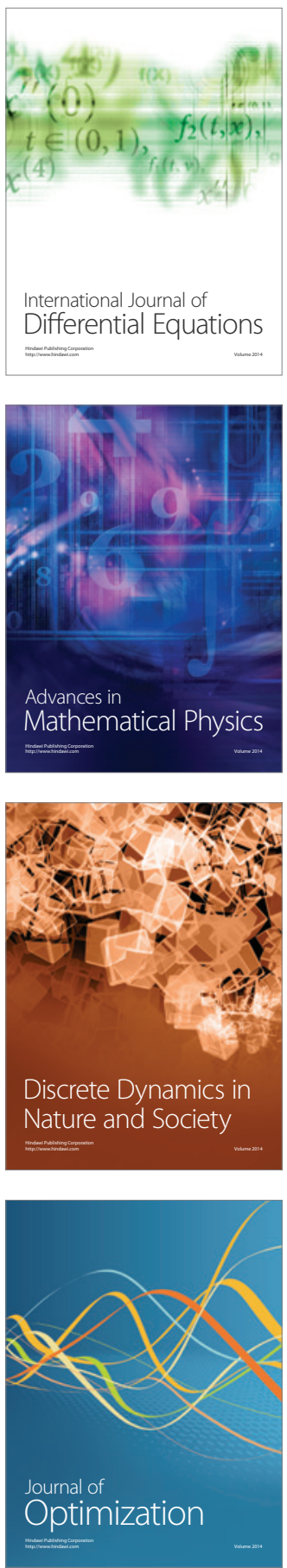